\title{
X-ray quasi-periodic pulsations in solar flares as magnetohydrodynamic oscillations
}

\author{
C. Foullon ${ }^{1}$, E. Verwichte ${ }^{1}$, V. M. Nakariakov ${ }^{1}$, and L. Fletcher ${ }^{2}$ \\ 1 Department of Physics, University of Warwick, Coventry CV4 7AL, UK \\ e-mail: foullonc@astro.warwick.ac.uk \\ 2 Department of Physics and Astronomy, University of Glasgow, Glasgow G12 8QQ, UK
}

Received 21 June 2005 / Accepted 2 August 2005

\begin{abstract}
We report the first observation at high spatial resolution of long-period quasi-periodic pulsations (QPP) of X-ray radiation during solar flares, made possible with the Reuven Ramaty High Energy Solar Spectroscopic Imager (RHESSI), supported by complementary data at other wavelengths from space-based and ground-based telescopes. Evidence for the presence of a transequatorial loop possibly responsible for the detected periodicity connected with its kink mode is found. Our findings suggest that QPP can be interpreted as a periodic pumping of electrons in a compact flaring loop, modulated by oscillations in a magnetically linked and larger loop acting as a long-period magnetohydrodynamic resonator.
\end{abstract}

Key words. Sun: corona - flares - oscillations - X-rays, gamma rays

\section{Introduction}

Quasi-periodic pulsations (QPP) of flaring emission, with periods from tenths of seconds to several minutes, have been detected since the early $70 \mathrm{~s}$ at wavelengths covering a wide frequency band from radio waves to hard X-rays (Aschwanden 2004) and were suggested to be produced by magnetohydrodynamic (MHD) waves (e.g. Roberts et al. 1983, 1984). Lowfrequency variations, with periods of several minutes, are of particular relevance to remote diagnostics of solar plasmas (Nakariakov et al. 1999) and to the interpretation of similar phenomena in stellar flares (Mathioudakis et al. 2003) where no spatial resolution is available.

Low-frequency variations in solar X-ray emission are not well reported and are not well understood. Imaging observations of such QPP were obtained in the 80s but had poor spatial resolution: the coarse $3.5-5.5 \mathrm{keV}$ images from the Hard X-ray Imaging Spectrometer on the Solar Maximum Mission indicated that long periods $(12-29 \mathrm{~min})$ were seen to originate at the foot of, or below, large-scale coronal loops (Švestka et al. 1982; Harrison 1987), providing a basis for the interpretation of similar oscillations in the 0.5-4.0 $\AA$ channel data of GOES-2 (Švestka et al. 1982; Švestka 1994). Similar periods have been observed recently off the solar limb, in 27 flare-like events, with periods between 7 and $31 \mathrm{~min}$, in the coronal emission lines $\mathrm{Fe}$ XIX (6.3 MK) and Fe XXI (8.9 MK) from the Solar Ultraviolet Measurements of Emitted Radiation (SUMER) spectrograph on the Solar an Heliospheric Observatory (SOHO) (Wang et al. 2003a,b, 2005). Here with RHESSI (Lin et al. 2002), we investigate, on a qualitatively new observational level, the presence of long-period variations observed in X-rays.

\section{Observations}

We study the flaring activity of February 5-6, 2003. We observe in full disk measurements with GOES-8 and RHESSI satellites two sequences of QPP, taking place less than $5 \mathrm{~h}$ apart (between 21:00 UT on the 5th and 3:00 UT on the 6th; see Figs. 1a and b). The QPP are well seen in X-rays at energies between 3 and $25 \mathrm{keV}$. Imaging facilities provided by RHESSI are supported by imaging data from the Soft X-ray Imager (SXI/GOES-12) and the Extreme ultraviolet Imaging Telescope (EIT/SOHO), magnetograms from the Michelson Doppler Imager (MDI/SOHO), and $\mathrm{H} \alpha$ images from the Big Bear Solar Observatory (BBSO) and the Learmonth Observatory. We find that two principal active sources are present in all images: a region denoted A, situated in NOAA Active Region 10277 in the southern hemisphere, and a region denoted B, situated in NOAA Active Region 10278 in the northern hemisphere (see Fig. 2).

The first sequence of QPP on February 5, denoted "QPP1", is associated with two flares: as seen by GOES, the first flare of class C2.1 peaks at 20:37 UT and the second flare of class C2.2 at 21:39 UT. The second sequence on February 6, denoted "QPP2", follows a C3.4 flare peaking at 2:12 UT. In each sequence, RHESSI and SXI imaging data are averaged over region A and over region B. Because of their lower timeresolution, the SXI lightcurves are primarily used to indicate the sites of flaring activity, in particular during RHESSI orbit 

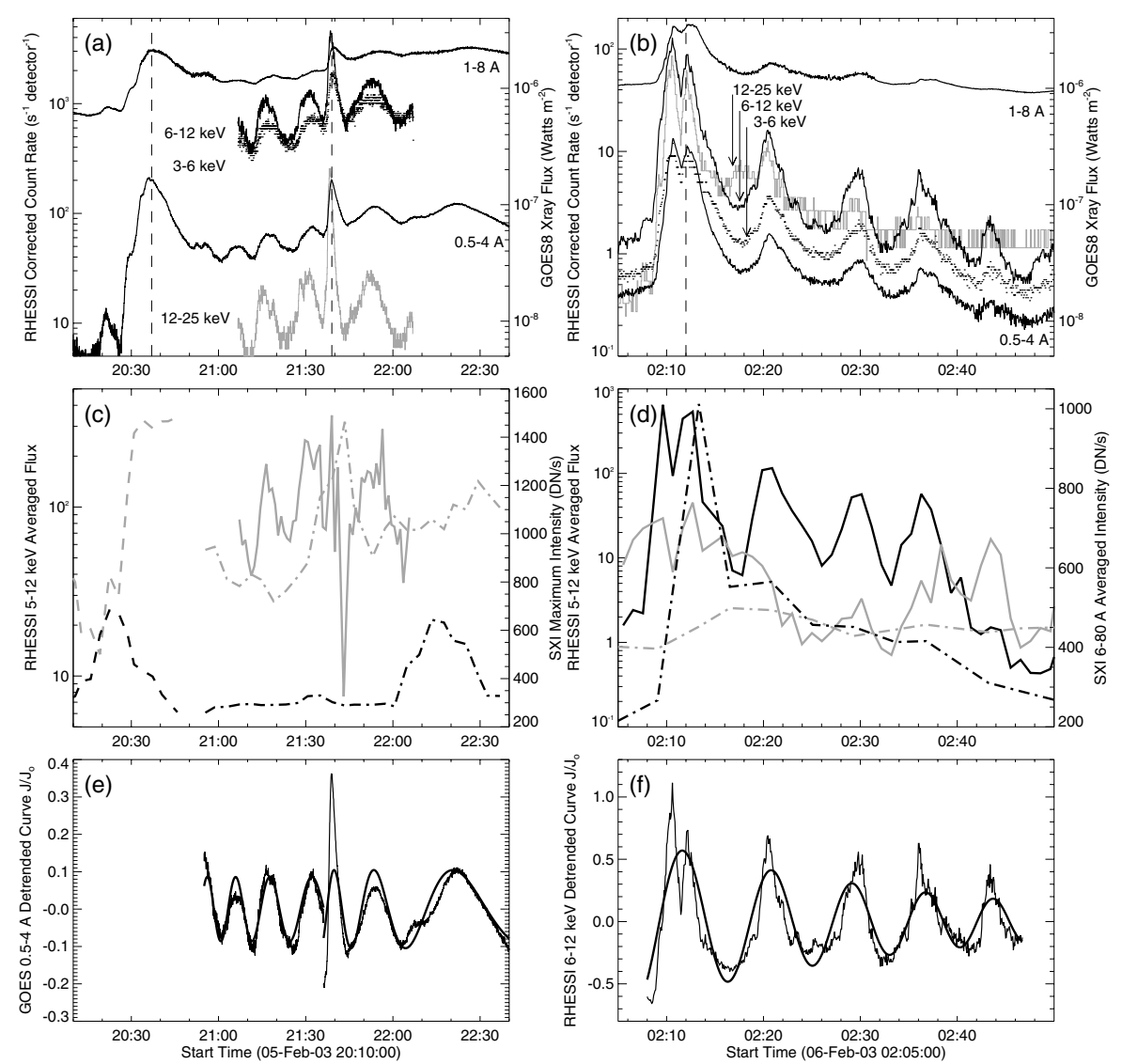

Fig. 1. QPP on February 5-6 2003. a) b) Full disk measurements in X-rays from GOES-8 (1-8 $\AA$ and 0.5-4 $\mathrm{A}$ ) and RHESSI (3-6 keV, 6-12 keV and 12-25 keV). The vertical dashed lines indicate the peak times of the flares, as seen by GOES 1-8 $\AA$. c), d) RHESSI averaged flux and SXI intensity in region A (black) and B (grey). RHESSI curves, shown as solid lines, are averaged flux above $80 \%$ of the maximum value in 5-12 keV RHESSI images reconstructed from collimators $1 \mathrm{~F}$ and $3 \mathrm{~F}-6 \mathrm{~F}$. The SXI curves are shown as dot-dashed lines in the Open filter $(6-80 \AA$ with short integration time of $0.0155 \mathrm{~s}$ ) and as dashed lines in the Thin Polyimide filter (6-60 § with integration time of $0.3345 \mathrm{~s}$; scales are arbitrary). e), f) Fits, shown as solid thick lines, for the detrended curves $J / J_{0}$, where e) $J^{2}$ is the GOES $0.5-4 \AA$ curve and $J_{0}$ is a linear trend; f) $J^{2}$ is the RHESSI 6-12 keV curve and $J_{\mathrm{o}}$ is the residual in a wavelet à trous decomposition (Starck \& Murtagh 2002). This latter method decomposes a time series in a sequence of approximations of the signal that have increasing time scales. The trend $J_{0}$ obtained was found to be more appropriate than a linear trend for the type of time series studied, and is smoother than a running average. night. As shown in Figs. 1c and d, we find that QPP are not a collection of flares at different locations: each sequence of QPP originate from one single site, which corresponds to the site of the associated flare(s). But the QPP do not all originate from the same region: QPP1 originate from region $\mathrm{B}$, whereas QPP2 originate from region A. Moreover, as QPP originate from a given active region, aperiodic activity occurs in the region oppositely located across the solar equator. This scenario is observed in the two occasions less than $5 \mathrm{~h}$ apart, with the same regions exchanging roles.

In Fig. 3, we have coaligned the RHESSI reconstructed images with the supporting images. RHESSI images are coaligned with respect to EIT and MDI images with an accuracy better than 1.6 arcsec (given that the pointing accuracies of RHESSI and SOHO are better than 0.6 and 1 arcsec respectively). $\mathrm{H} \alpha$ images are coaligned with respect to MDI images with an accuracy better than 1 MDI pixel, i.e. 2 arcsecs. From the coalignments, we infer that in both regions, the emission in X-ray originates from the top of a small loop: the upper body of the small loops are seen in the EUV with EIT (195 $\AA)$; the MDI magnetograms and the $\mathrm{H} \alpha$ images are complementary to indicate the footpoint locations. The location of the soft X-ray emission is thus consistent with a thermal interpretation.

The periodicities in QPP1 and QPP2 are similar and of the order of 8-12 min. To characterise more precisely the periodicities in the X-ray curves, $I(t)$ at time $t$, it is useful to define the time series as $J(t)=\sqrt{I(t)}$, assuming thermal emission of the form $I \sim n_{\mathrm{e}}^{2}$ where $n_{\mathrm{e}}$ is the electron density. We observe that the pulsations in QPP1 are interrupted by the second flare. As seen by RHESSI, this second flare occurs slightly

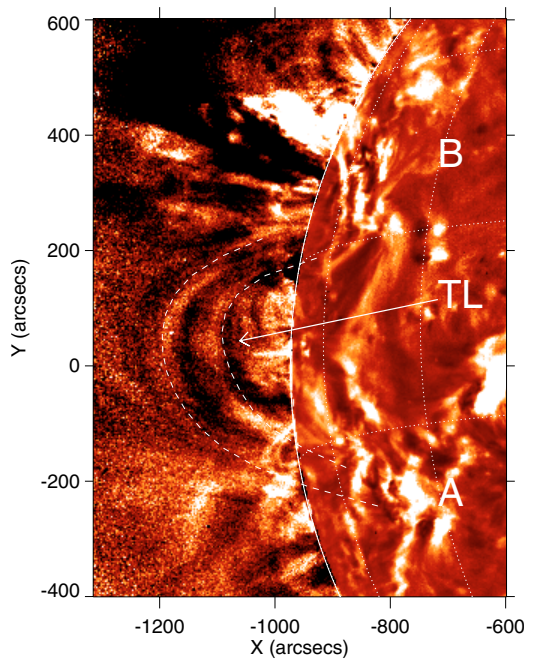

Fig. 2. Difference of EIT $195 \AA$ images taken on February 2003, at 21:36 UT on the 5th and 02:12 UT on the 6th. The two principal active sources are denoted "A" and "B" and the transequatorial loop, shown between dashed lines, "TL". The contrast in the off-disk area is enhanced using histogram-equalisation.

to the South-East of the pulsating loop-top-source, in a neighbouring loop seen as the second bright loop-top in the EIT image in Fig. 3a. Using the GOES 0.5-4 $\AA$ curve (instead of a RHESSI curve interrupted by the RHESSI orbit night), we study separately the part before the interruption and the following part including the flare. For each part, the detrended curves are fitted with a cosine curve $F(t)$ with linear period change, viz. $F(t)=A \cos \left(2 \pi t /\left(P_{\mathrm{o}}+\lambda t\right)-\phi\right)$, for amplitude $A$, initial 


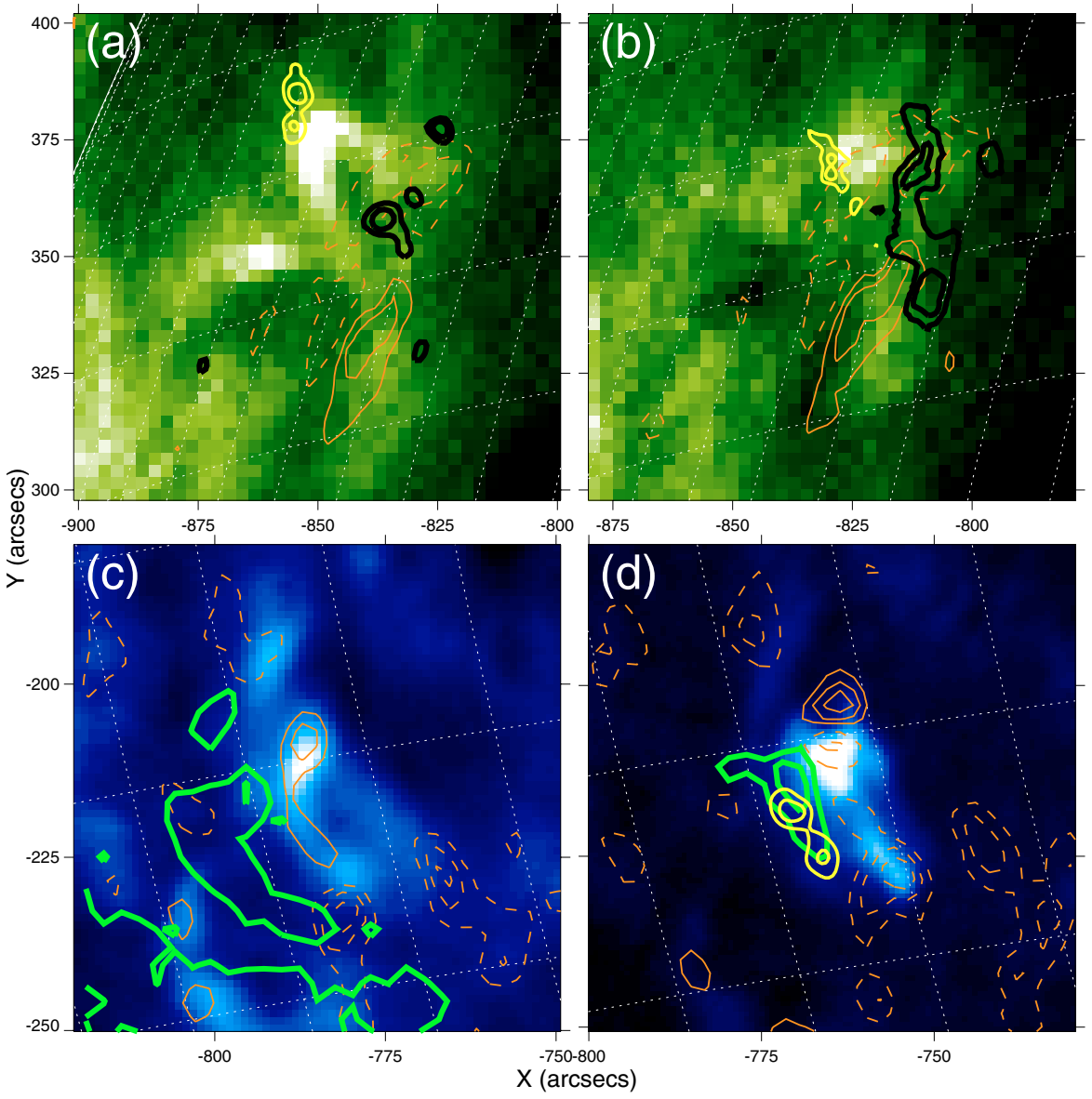

Fig. 3. a), b) EIT $195 \AA$ images of region B and c), d) $\mathrm{H} \alpha$ images of region $\mathrm{A}$ at the time of the first and second sequence of QPP. Superimposed are the contour levels of: 5-12 keV RHESSI images (reconstructed from collimators $1 \mathrm{~F}$ and $3 \mathrm{~F}-6 \mathrm{~F}$ ) shown in solid lines (at 50 and $80 \%$ of the maximum value), the longitudinal magnetic field observed by MDI in thin lines (solid for positive field and dashed for negative field), $\mathrm{H} \alpha$ images in thick black (80 and 90\%, in a) and b)) and EIT $195 \AA$ images in thick solid lines (50 and $80 \%$, in c) and d)). a), c) Images taken on 5 February 2003 are co-aligned at 21:52:18 UT. This includes an $\mathrm{H} \alpha$ image from BBSO (21:43:57 UT), images by EIT (21:47:32 UT), MDI (20:53 UT, with contour levels multiple of $2 \times 10^{-2}$ Tesla-equivalent) and a RHESSI image (in the time range 21:51:4721:52:49 UT, shown in a)). b), d) Images taken on 6 February 2003 are co-aligned at 2:20:54 UT. This includes $\mathrm{H} \alpha$ images from Learmonth (2:27:44 UT in b), 2:16:39 UT in d)), images by EIT (2:22:48 UT), MDI (3:17 UT, with contour levels multiple of $10^{-2}$ Tesla-equivalent), and RHESSI images (in the time range 02:17:19-02:18:20 UT in b) and 2:20:23-2:21:24 UT in d)). period $P_{\mathrm{o}}$, period change rate $\lambda$ and phase $\phi$. This is the simplest function that best characterises the time series, as illustrated in Fig. 1e. Prior to the flare interruption, periodicities increase from $P_{\mathrm{o}}=8.74$ to $12.21 \mathrm{~min}(\lambda=0.0846$, over the duration $\Delta t=41.1 \mathrm{~min}$ of the time series). In the second phase, the periods increase much faster, starting with the flare from $P_{\mathrm{o}}=8.59 \mathrm{~min}$ and broadening up to $23.06 \mathrm{~min}(\lambda=0.2267$, $\Delta t=63.9 \mathrm{~min})$. For QPP2, the detrended curve is fitted with a cosine curve $G(t)$ with linear period change and exponential decay, viz. $G(t)=F(t) \exp \left(-t / \tau_{\mathrm{d}}\right)$, for damping time $\tau_{\mathrm{d}}$. In Fig. 1f, the fit to the RHESSI 6-12 keV curve shows a slight decrease in $P$ from $P_{\mathrm{o}}=10$ to $8 \mathrm{~min}(\lambda=-0.0512, \Delta t=40 \mathrm{~min})$, with a decay time $\tau_{\mathrm{d}}=28.36 \pm 5.81 \mathrm{~min}$, which corresponds to a damping factor $\tau_{\mathrm{d}} / P_{\mathrm{o}}=2.84$.

\section{Discussion}

The quasi-periodic variations may be interpreted as some periodic pumping, or other energisation, of electrons in the flaring loop, modulated by MHD oscillations. The length-scale of the loop, though, cannot explain such long periods as the largest period of a wave that could be sustained by one of the flaring loops in either region $\mathrm{A}$ or region $\mathrm{B}$, the fundamental period of a slow magnetoacoustic standing mode, would not exceed $100 \mathrm{~s}$ (with a loop length of up to $22 \mathrm{Mm}$ and temperature of $10 \mathrm{MK}$ ). Therefore we suppose that the modulation of the flaring emission is due to MHD oscillations in a magnetically linked, larger resonating loop. In site $\mathrm{A}$ or $\mathrm{B}$, the footpoint of a long loop (resonator) is adjacent and may be connected to the smaller flaring loop (external exciter). A transequatorial coronal loop connecting the two regions is a possible resonator to explain the long periods. The presence of such a loop is strongly suggested by the sympathetic activity between regions A and B and the similarity of the periodicities found at the opposite sites. An arcade of loops connecting regions A and B can be seen in EIT images. One of these loops is shown in Fig. 2 within dashed lines. The northern footpoint of this arcade corresponds to a site at lower latitude than region $\mathrm{B}$, but is connected to the same AR 10278.

Taking the observed period $P$ and loop length $L$ of the transequatorial loop, and since the wavelength of a fundamental standing mode is twice the length of the loop, one can estimate the phase speed required as $V_{\mathrm{ph}}=2 L / P$. With a loop length estimated to be 500-600 Mm and periods found of 8-12 min, we find phase speeds between 1400 and $2500 \mathrm{~km} \mathrm{~s}^{-1}$. These phase speeds are consistent with the speeds of fast magnetoacoustic kink modes that have been directly observed in coronal loops with the Transition Region And Coronal Explorer (TRACE) instrument (Aschwanden et al. 1999; Nakariakov et al. 1999; Verwichte et al. 2004). In the present case, unfortunately, TRACE was observing a different region of the solar disk. The weak damping of the QPP is also consistent with the damping of the kink oscillations discovered with TRACE, whose decay factor $\tau_{\mathrm{d}} / P$ has been reported to vary between 1 and 4 . For QPP2, where $\tau_{\mathrm{d}} / P \sim 2.84$, the real decay time in the resonating loop could be much larger 
than the one observed. As for QPP1, there was no measurable decay. However, the lack of decay can be connected with some energy supply mechanism (see Verwichte et al. 2004).

If the observed modulations were caused by another MHD mode, the slow magnetoacoustic mode, then the transequatorial loop would need to have an unrealistic temperature for a quiescent coronal loop (see Nakariakov et al. 2004). It is conceivable that medium-size loops might be the acoustic resonators providing the periodicities in question. However the causal configuration in SUMER events, where the initial injection of hot flows at one footpoint from a magnetically connected small flaring loop excites the slow mode waves in a bigger loop (Wang et al. 2005), is the exact inverse of the present situation (i.e. something drives externally the small flaring loop). Besides, it is not clear how a basically longitudinal mode present in the large loop could modulate the emission of the smaller, flaring loop. This leaves the fast magnetoacoustic kink mode as the most likely candidate. This mode is an attractive option, as it perturbs the magnetic field lines in the transverse direction, and in turn may compress the field lines of the flaring loop. In turn, the periodic oscillations in magnetic field causes the plasma in the flaring loop to be successively compressed and expanded, modulating the particle acceleration process. Magnetic pumping (also called betatron acceleration) is one possible mechanism, which is governed by the conservation of the first adiabatic invariant, when the field changes slowly (Brown \& Hoyng 1975). This condition is met, as the observed periods $P$ are much greater than the selfcollision timescale $t_{\mathrm{c}}=2.7 \times 10^{5} T_{\mathrm{e}}^{3 / 2} /\left(\ln \Lambda n_{\mathrm{e}}\right) \sim 4.4 \times 10^{-2} \mathrm{~s}$ (for Coulomb Logarithm $\ln \Lambda \sim 19.25$, electron temperature $T_{\mathrm{e}} \sim 10^{7} \mathrm{~K}$ and density $n_{\mathrm{e}} \sim 10^{16} \mathrm{~m}^{-3}$ ).

A loop kink mode is excited in response to a dynamic event, such as flares, prominence eruptions or coronal mass ejections (CMEs) (Schrijver et al. 2002). The event may occur nearby or at some distant site. In the latter case, it is believed that a passing blast wave generated by the event perturbs the loop. The first flare on February 5 in AR 10278 is associated with a small filament eruption in the same region (starting at 20:24 UT, as seen by EIT and in $\mathrm{H} \alpha$ images) and a corresponding CME. The second event on February 6 in AR 10277 is followed by a filament eruption in the same region around 3:00 UT (as seen by EIT and in H $\alpha$ images) and an M1.2 flare in AR 10278, peaking at 3:49 UT and associated with a CME. In both cases, a cavity and some filament-like material followed the wide loop front of the CME. The monotonic change of the periods in the QPP might be accounted for by changes in the coronal environment immediately after or before the eruptions. An increase in period would either indicate a decrease in magnetic field strength, an increase in density or an increase in oscillating loop length, and vice-versa. Any of these changes in the transequatorial loop associated with a large-scale coronal eruption are plausible.

Therefore the assumption that the long periods of QPP can be explained in terms of MHD modes proves to be remarkably robust. The new evidence of QPP that we have investigated, based upon the novel observational opportunities provided by RHESSI, demonstrate that flare-induced long-period QPP can be explained as modulations of emission in a small flaring loop due to fast kink MHD waves in a magnetically linked and large-scale loop. The nature of periodic pumping of electrons in the flaring loops will be addressed in a future detailed multi-wavelength analysis of the spatially-resolved QPP.

For the first time observationally, we have linked together solar flare pulsations and a MHD kink oscillation that has been widely exploited in recent years through MHD seismology (Nakariakov \& Verwichte 2005). However, in the absence of spectroscopic and high-cadence EUV imaging data, we had to draw conclusions indirectly, based upon the observed loop structures, oscillation characteristics, wave theory and phenomenology. As a result other interpretations involving in particular the slow standing MHD mode cannot be entirely ruled out. This study marks the beginning of a wealth of opportunities to resolve QPP on the solar disk with RHESSI. Combined with complementary data at other wavelengths, in particular high-resolution EUV images from instruments such as TRACE or the EUV Imaging Spectrometer (EIS) on the future Solar-B mission, such opportunities have the potential to provide remote diagnostics of solar plasma and broaden our understanding of physical processes operating in solar and stellar flares.

Acknowledgements. We gratefully acknowledge the open-data policies of RHESSI and GOES. EIT and MDI data are courtesy of SOHO consortia; SOHO is a project of international cooperation between ESA and NASA. H $\alpha$ images are courtesy of BBSO, operated by the NJIT, and SOONSPOT with the kind assistance of A. Kiplinger.

\section{References}

Aschwanden, M. J. 2004, Physics of the Solar Corona (SpringerPraxis)

Aschwanden, M. J., Fletcher, L., Schrijver, C. J., \& Alexander, D. 1999, ApJ, 520, 880

Brown, J. C., \& Hoyng, P. 1975, ApJ, 200, 734

Harrison, R. A. 1987, A\&A, 182, 337

Lin, R. P., Dennis, B. R., Hurford, G. J., et al. 2002, Sol. Phys., 210, 3 Mathioudakis, M., Seiradakis, J. H., Williams, D. R., et al. 2003, A\&A, 403, 1101

Nakariakov, V. M., Ofman, L., Deluca, E. E., Roberts, B., \& Davila, J. M. 1999, Science, 285, 862

Nakariakov, V. M., Tsiklauri, D., Kelly, A., Arber, T. D., \& Aschwanden, M. J. 2004, A\&A, 414, L25

Nakariakov, V. M., \& Verwichte, E. 2005, Living Rev. Solar Phys., 2, 3, URL (2005/07/05)

http://www.livingreviews.org/lrsp-2005-3

Roberts, B., Edwin, P. M., \& Benz, A. O. 1983, Nature, 305, 688

Roberts, B., Edwin, P. M., \& Benz, A. O. 1984, ApJ, 279, 857

Schrijver, C. J., Aschwanden, M. J., \& Title, A. M. 2002, Sol. Phys., 206, 69

Starck, J., \& Murtagh, F. 2002, Astronomical image and data analysis (Astron. and Astrophys. Library Berlin: Springer)

Švestka, Z. 1994, Sol. Phys., 152, 505

Švestka, Z., Dennis, B. R., Woodgate, B. E., et al. 1982, Sol. Phys., 80,143

Verwichte, E., Nakariakov, V. M., Ofman, L., \& Deluca, E. E. 2004, Sol. Phys., 223, 77

Wang, T. J., Solanki, S. K., Curdt, W., et al. 2003a, A\&A, 406, 1105

Wang, T. J., Solanki, S. K., Innes, D. E., Curdt, W., \& Marsch, E. 2003b, A\&A, 402, L17

Wang, T. J., Solanki, S. K., Innes, D. E., \& Curdt, W. 2005, A\&A, 435, 753 